\title{
Anal Papanicolaou Smear in Women with Abnormal Cytology: a Thai Hospital Experience
}

\author{
Panya Sananpanichkul ${ }^{1 *}$, Sirida Pittyanont ${ }^{1}$, Prapap Yuthavisuthi ${ }^{1}$, Nutchanok \\ Thawonwong ${ }^{2}$, Malee Techapornroong ${ }^{3}$, Kornkarn Bhamarapravatana ${ }^{4}$, Komsun $^{2}$ \\ Suwannarurk ${ }^{5,6}$
}

\begin{abstract}
Background: Anal intraepithelial lesions (AIL) are likely to represent a precursor for anal cancer. Women infected with human immunodeficiency virus (HIV) may be at higher risk of anal cancer but a screening program for AIL still is not routinely recommended. We here studied the relationship of dysplastic cells from cervical and anal cytology in HIV-infected women. Materials and Methods: This prospective study was conducted in Prapokklao Hospital, Thailand during 2013-2014. Five hundred and ninety nine HIV-infected women were recruited. Participants who had cytological reports of equally or over "abnormal squamous/glandular cells of undetermined significance" (ASC-US) were classified as abnormal cervical or anal cytology. Descriptive statistics and logistic regression analysis were used to evaluate correlations between groups. Results: HIV-infected women with abnormal cervical cytology had 3.8 times more risk (adjusted odd ratio 3.846, 95\% confidence interval 1.247-11.862, p-value. 019) for abnormal anal cytology. The major problem of the anal Pap test in this study was the inadequacy of the collected specimens for evaluation $(34.4 \%, 206 / 599)$. Sensitivity, specificity, positive predictive value, negative predictive value and accuracy of cervical and anal Pap tests were 93.9/12.0, 87.3/96.9, 39.7/21.4,99.4/94.1 and 88.1/91.4 percent, respectively. Conclusions: Abnormal cervical cytology in HIV-infected women indicates elevated risk for abnormal anal cytology. The sensitivity of the anal Pap test for detection of AIL 2/3 in HIV-infected women was quite low while specificity was excellent. Inadequacy of specimen collection for evaluation was a major limitation. Improvement of sample collection is recommended for future investigations.
\end{abstract}

Keywords: Papanicolaou Smear - pap test - anus - cervix - HIV - CIN - AIN

Asian Pac J Cancer Prev, 16 (3), 1289-1293

\section{Introduction}

Anal cancer is a relatively uncommon malignancy. Since the anorectal transitional zone shares an embryonic origin with the uterine cervix, human papillomavirus (HPV) infection is also a major cause of anal cancer as well as cervical cancer. Other known risk factors are human immunodeficiency virus (HIV) infection with low cluster of differentiation 4 (CD4) count, anoreceptive intercourse, cigarette smoking (Etienney et al., 2008; Baranoski et al., 2012), frequent sexuality, uncircumcised partner and other HPV-related cancers such as women with cervical cancer, vaginal cancer, or vulvar cancer (American Cancer Society, 2014). Additionally, women with HIV infection had more than 5 and 14 times to develop cervical and anal cancer comparing to the general population, respectively (Chaturvedi et al., 2009).

Anal cancer developed from squamous intraepithelial lesion (SIL) the same as cervical cancer. Anal SIL or anal intraepithelial neoplasia (AIN) was categorized to low and high grade. Low grade AIN was likely to rapidly progress to high grade AIN (Palefsky et al., 1998). In several cases with biopsy-proven SIL, atypical squamous cells of undetermined significance (ASC-US) may also be detected by anal cytological examination (Gaisa et al., 2014).

According to the World Bank data, there was $1.1 \%$ population with HIV infection in Thailand in 2013. The prevalence of abnormal anal Pap test in HIV-infected women in our institute is $2.2 \%$ (Pittyanont et al., 2014). As a result, anal cancer screening program could help to prevent anal cancer in a high-risk population. Unfortunately, there was no routine anal cytology screening program implemented in any Thai hospitals. The objective of this study is to investigate the relationship of cervical and anal cytological screening in HIV-infected women who attended gynecological clinic.

${ }^{1}$ Department of Obstetrics and Gynaecology, ${ }^{2}$ Department of Pathology, ${ }^{3}$ Department of Medicine, Prapokklao Hospital, Chanthaburi, ${ }^{4}$ Department of Preclinical Science, ${ }^{5}$ Department of Obstetrics and Gynaecology, ${ }^{6}$ Chulabhorn International College of Medicine, Thammasat University, Pathum Thani, Thailand*For correspondence: panysanan@yahoo.com 


\section{Materials and Methods}

This was a descriptive prospective study. Correlation study of dysplastic cells between cervical and anal Pap test in HIV-infected women was performed include of their risk factors. After the study was approved by Prapokklao Hospital Ethics committee, data were collected from HIV-infected women who came for a routine checkup at Prapokklao Hospital HIV-Gynaecological Clinic, Chanthaburi province, Thailand. Period of study was from March 2013 to February 2014. All participants volunteered in this study have signed written consent forms. Then, each individual was given a self-administered questionnaire. Those who could not read proficiently were personally interviewed by the physicians to help them answering the questionnaires.

All women with confirmed diagnosis of HIV infection who visited our HIV-Gynaecological Clinic during the study period were included in this study. Participants with active lower gastrointestinal bleeding, anal inflammation, acute anal fissure and those who did not want to participate were excluded.

Anal Pap test was collected using a long side of modified Ayre's spatula (Chaves et al., 2012). The spatula was inserted into the anal canal for $2 \mathrm{~cm}$. then rotated 360 degree in a conical motion and removed. Sample was smeared onto a glass slide and immediately fixed in $95 \%$ ethyl alcohol for 15 minutes. Air dried samples were transported to the laboratory and reviewed by cytopathologists.

Anal cytological results were classified as satisfactory for evaluation, negative for intraepithelial lesion or malignancy (NILM), atypical squamous cells of undetermined significance (ASC-US), low grade squamous intraepithelial lesion (LSIL), high grade squamous intraepithelial lesion (HSIL) and cancer. Evaluation criteria for cervical cytology used in this study were followed 2001 Bethesda System Terminology (Solomon et al., 2004). Women with abnormal cervical cytology were sent for colposcopic evaluation and biopsy was taken if indicated.

Women who had abnormal anal cytologic results of equal to, or over "abnormal squamous/glandular cells of undetermined significance" (ASC-US or AGUS) were contacted for further HPV testing. HPV testing was performed using a commercially available kit of Cervista human papillomavirus high risk (HPV HR) assays (Hologic, Inc, Marlborough, MA, USA). If HPV HR testing showed positive for high risk HPV, the second step of HPV testing using Cervista HPV 16/18 assays kit (Hologic, Inc, Marlborough, MA, USA) was performed. This HPV testing method is analyzed by polymerase chain reaction (PCR) techniques according to the manufacturer's guidelines. Subsequently, the patients were referred to the department of surgery (colorectal) for anoscopy and biopsy if indicated. They were further followed-up by surgeons in department of surgery if biopsy results showed anal dysplasia

Statistical analysis was performed using Statistical Package for the Social Sciences (SPSS) version 19.0
(SPSS, Chicago, IL, USA). Frequency, percentage and the average mean were represented according to demographic characteristics. Chi-square test was used to compare categorical data. Logistic regression analysis was used to evaluate the correlation between both groups and p-value of less than 0.05 was defined as statistical significance.

\section{Results}

In this study, 599 participants were enrolled. All were HIV-infected patients who came for routine followup program at Prapokklao Hospital. Mean age of the participants was $40.9 \pm 8.9$ years with the range of 19 to 70 years old. Ninety percent of them were older than 30 years old. Majority worked for local businesses and 30.7 percent of them worked in agriculture and proprietary trade. Only 5 percent of the participants had undergraduate degrees while the rest of them were primary or secondary school graduates. Multiparous participants were the largest group of the population. Condom was used in 64.9 percent of the participants and homosexual behavior was accounted for 1.4 percent (8/599). Alcohol consumption and smoking habit were found only 3 and 1.7 percent, respectively. Most of the participants had CD4 count more than 200 per $\mathrm{mm} 3$. Ninety five percent of them had no history of other sexual transmitted diseases. A comparison of demographic and characteristic data with reference to cervical and anal cytology is presented in Table 1.

Inadequacy of the collected specimen for evaluation was $34.4 \%$ (206/599). After exclusion of cases with low-quality samples Pap test, three hundred and ninety three patients were still participated in the study. Table 1 showed characteristics associated with abnormal anal Pap test comparing to negative anal Pap test. Duration of seropositive, condom usage and abnormal cervical Pap test were statistically significant when associated with abnormal anal Pap test with p-value at 0.002, 0.045 and less than 0.001 , respectively. Participants' age, occupation, education, family status, history of sexual transmitted diseases, smoking habit, alcohol consumption and CD4 count had no significant correlation to the occurrence of abnormal anal Pap test.

The prevalence of abnormal cervical and anal Pap test of HIV-infected women in this study were 19.4 $(116 / 599)$ and $2.3(14 / 599)$ percent. Table 2 shows data for sensitivity, specificity, positive predictive value (PPV), negative predictive value (NPV) and accuracy of cervical and anal Pap test which were found to be 93.9/12.0, 87.3/96.9, 39.7/21.4, 99.4/94.1 and 88.1/91.4 percent, respectively.

Table 3 presented the logistic regression analysis of the characteristics that associated with abnormal anal Pap test. HIV-infected participants with abnormal cervical Pap test were more likely to have abnormal anal Pap test (odds ratio [OR] 3.846, 95\% confidence interval [CI] 1.247-11.862, $\mathrm{p}=0.019$ ). Duration of seropositive also showed significant association in contrast to the effect of cervical Pap test. HIV-infected participants with short seropositive duration were more likely to have abnormal anal Pap test (OR 0.704, 95\%CI 0.534-0.929, $\mathrm{p}=0.013$ ). 
Table 1. Demographic Characteristics and Prevalence of Abnormal Cervical Comparing to Anal Cytology in HIV Infected Women at Prapokklao Hospital, Chanthaburi, Thailand

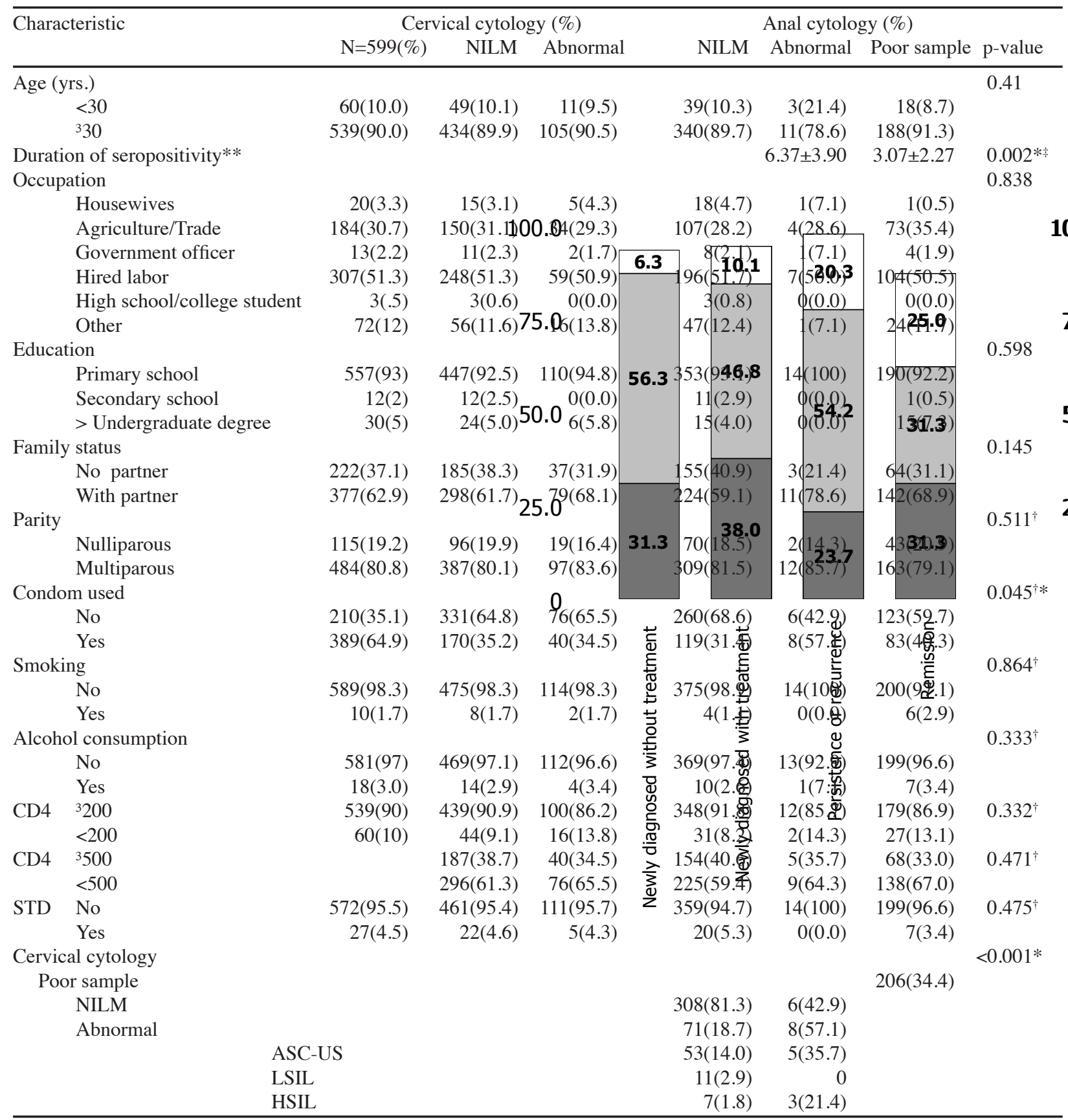

†Fisher's Exact Test; *statistic significant; **(mean \pm SD), ${ }^{*}$ t-test, CD4: cluster of differentiation 4, STD: sexual transmitted disease; NILM: negative for intraepithelial lesion or malignancy, ASC-US: atypical squamous cells of undetermined significance, LSIL: low grade squamous intraepithelial lesion, HSIL: high grade squamous intraepithelial lesion, CD4: cluster of differentiation 4, STD: sexual transmitted disease

Table 2. Comparison of Cervical and Anal Cytology for Detection of Squamous Intraepithelial Neoplasia 2/3

\begin{tabular}{lcccc}
\hline & \multicolumn{2}{c}{ Cervical } & \multicolumn{2}{c}{ Anal } \\
Cytology & CIN2/3 & $<$ CIN 1 & AIN2/3 & <AIN 1 \\
\hline Positive & 46 & 70 & 3 & 11 \\
Negative & 3 & 480 & 22 & 349 \\
Sensitivity (95\%CI) & $93.9(83.11-98.65)$ & $12.0(2.69-31.25)$ \\
Specificity (95\%CI) & $87.3(84.19-89.94)$ & $96.9(94.60-98.46)$ \\
PPV (95\%CI) & $39.7(30.69-49.16)$ & $21.4(4.91-50.79)$ \\
NPV (95\%CI) & $99.4(98.19-99.86)$ & $94.1(91.16-96.25)$ \\
Accuracy (\%) & 88.1 & 91.4 & \\
\hline
\end{tabular}

*CIN: cervical intraepithelial neoplasia, AIN: anal intraepithelial neoplasia, CI: confidential interval; PPV: positive predictive value, NPV: negative predictive value
Table 3. Adjusted Odd Ratio of Characteristic Associated with Abnormal anal Cytology

\begin{tabular}{lclc}
\hline Characteristic & Adjusted OR & \multicolumn{1}{c}{$95 \%$ CI } & p value \\
\hline Cervical cytology & 3.846 & $1.247-11.862$ & $0.019^{*}$ \\
Duration of seropositive & 0.704 & $0.534-.929$ & $0.013^{*}$ \\
Condom used-family & 0.38 & $0.124-1.167$ & 0.091 \\
planning & & & \\
\hline
\end{tabular}

*significant p-value $<0.05$; Adjusted OR: adjusted odd ratio, CI: confidential interval

Condom usage (for family planning purpose) did not have any significant effect to abnormal anal Pap test (OR 0.38, 95\%CI 0.124-1.167, $\mathrm{p}=0.091$ ). 


\section{Discussion}

Anal cancer is a rare disease with $0.3 \%$ increase in incidence per year from 2004 through 2008 (Siegel et al., 2012). Known risk factors of anal cancer include HPV infection (Ghosh et al., 2012; Afshar et al., 2013), HIV infection with low CD4 count, anoreceptive intercourse and cigarette smoking (Baranoski et al., 2012; Zhang et al., 2012). When concerned about cervical cancer screening, Pap test is widely accepted as an effective screening tool for cervical precancerous lesion and cancer. It significantly helps lowering the mortality from cervical cancer. However, colposcopic-directed biopsy is still the gold standard for cervical cancer diagnosis in women with abnormal cervical cytology. In the USA, there is still no definite guideline or recommendation on anal cytologic screening offered for AIN which may progress to anal cancer (MMWR Recommendations and Reports, 2009). Currently, there is also no anal cancer screening program in Thailand.

Human immunodeficiency virus (HIV) infection with low CD4 count, anoreceptive intercourse, cigarette smoking (Etienney et al., 2008; Baranoski et al., 2012), frequent sexuality, uncircumcised partner, number of sexual partner and other HPV-related cancer such as women with cervical cancer, vaginal cancer, or vulvar cancer (Centers for Disease Control and Prevention, 2014) were reported to be risk factors for anal cancer. People with lower nadir CD4 counts appear to be at higher risk of anal cancer (Bertisch et al., 2013). This was the same as in this study when the risks of abnormal anal cytology were concerned. In this study, the duration of seropositive, condom usage and abnormal cervical cytology had significant relation to the occurrence of abnormal anal cytology. The CD4 count, smoking habit and alcohol consumption did not show any significant effect to abnormal anal cytology.

Anoreceptive intercourse was found in 36-42\% of HIVinfected women in the United States (Conley et al., 2010; Kojic et al., 2011). In our previous study, the prevalence of anoreceptive intercourse is only $0.7 \%$ which is fairly low (Pittyanont et al., 2014). Since, by culture, Thai people are timid to talk about sexual subject (Suwannarurk et al., 2009), acceptance of culturally non-approved sexual conduct such as anoreceptive intercourse may be denied. Other than immune-compromised condition such as HIV infection, oncogenic HPV serotypes especially HPV16 , plays an important role in anal cancer development (Centers for Disease Control and Prevention, 2014).

$\mathrm{HIV}$-infected women have more than 5 times higher risk to have cervical cancer (standardized incidence ratio [SIR] 5.6) and more than 13-14 times higher risk to have anal cancer than general (SIR 13.1-14.5) (Piketty et al., 2012). Tissue around anal region is structurally similar to that of cervicovaginal region. Therefore, abnormal cervical cytology plays an important role for development of abnormal anal cytology. Our study suggests that HIVinfected women with abnormal cervical cytology had 3.8 higher risk to have abnormal anal cytology. Our result is consistent with the previous study by Lamme et al. (2014). High-grade AIN was also likely to be detected in anal cytology from HIV-infected individuals as well (Mullins et al., 2013).

Presently, anal cytology is still used by most to screen for anal cancer. But because of low sensitivity made it's not suitable for screening tool. Ferraris and coworker reported a low specificity and low sensitivity for Pap test in detecting abnormal anal cancer. It is imprudent to terminate a close anoscopic surveillance in high-risk patients based on only the normal Pap test result (Ferraris et al., 2008). In this study, the sensitivity of anal Pap test was only $12.0 \%$. This was different from $93.9 \%$ sensitivity of cervical Pap test. Fortunately, the anal Pap test had high specificity and high negative predictive value $(96.9 \%$ and $94.1 \%$, respectively) which could be used as diseaseexclusion tool. Since $34.4 \%$ of the samples collected in this study had low quality (206/599), improvement of specimen collection should be concerned. Anal Pap test is not routinely offered in Thailand. This result might be from the number of poor sample, however the specificity of the screening test was good. Stool softener or enema introduced before the specimen collection and modification the shape of spatula might be in consideration for next study.

Anal HPV also correlated with abnormal anal cytology. Our study supported the correlation of cervical and anal HPV infection as previously reported (Lamme et al., 2014). Previous study indicated that women with highrisk cervical HPV were more likely to have high-risk anal HPV with odds ratio of 3.6. Additionally, women with high-risk anal HPV were more likely to have abnormal anal cytology with odds ratio of 6.5. Although this study had a higher number of participants, there were more cases of unsatisfied Pap test when compared to the previous study(206[34.4\%]: 29[14.8\%]). Another contributing factor was the number of HIV-infected cases that in this study, we include only HIV-infected women not normal participants.

In this study, a fair numbers of abnormal anal cytology was detected. However, the report of anoreceptive intercourse in Thai women is fairly low. This might be from Thai culture and patients' embarrassment to reveal the practice of intercourse. In 2013, the number of adult Thai men with HIV was 257,293, with 5,899 new infected cases. This number was higher than women data. Although HIV prevalence among male sex workers declined from $16.0 \%$ in 2010 to $12.2 \%$ in 2012 , it remained significantly $19 \%$ higher than female sex workers and $20 \%$ higher than general men who have sex with men (MSM) population (Thai National Aids Committee, 2014). HIV prevalence among MSM is still high, especially among those living in large urban areas and international tourist destinations (e.g., Bangkok, Chiang Mai, Phuket, Pattaya). Therefore, anal cytology or anal HPV testing might be a promising screening tool for anal cancer especially in a high-risk population. A patient receptive system and protocol should be suggested in the future.

In conclusion, abnormal cervical cytology in HIVinfected women had 3.8 times higher risk for abnormal anal cytology while shorter duration of seropositive brought a higher risk for anal intraepithelial neoplasia. There is no standard recommendation for anal cancer 
screening in HIV-infected women. However the increasing incidence of the disease makes it a priority to initiate anal cancer screening standard protocol. Sensitivity of anal Pap test for detection of AIN2/3 is low though specificity is excellent. Inadequate specimen received for further evaluation is a major limitation. Improvement of sample collection is recommended for future investigation since anal cytological screening should be a routine service offering to both male and female population with high-risk HPV infection in the near future.

\section{Acknowledgements}

This work was funding supported by Research Institute of Prapokklao Hospital. The authors wish to thank Dr. Sinitdhorn Rujirabanjerd for her expert opinion.

\section{References}

Afshar RM, Mollaie HR, Fazlalipour M, et al (2013). Prevalence and type distribution of human papillomavirus infection using the INNo-Lipa assay, Kerman, Southeast Iran. Asian Pac J Cancer Prev, 14, 5287-91.

American Cancer Society (2014). Anal cancer. Causes, risk factors, and prevention topics. Atlanta, Ga: Am Cancer Soc.

Baranoski AS, Tandon R, Weinberg et al (2012). Risk factors for abnormal anal cytology over time in HIV-infected women. AM J Obstet Gynecol, 207, 107.

Bertisch B, Franceschi S, Lise M, et al (2013). Risk factors for anal cancer in persons infected with HIV: a nested case-control study in the Swiss HIV Cohort Study. Am J Epidemiol, 178, 877-84.

Centers for Disease Control and Prevention (2014). HPVAssociated Vulva Cancer Rates by Race and Ethnicity. Retrieved November 9.

Chaturvedi AK, Madeleine MM, Biggar RJ, et al (2009). Risk of human papillomavirus-associated cancers among persons with AIDS. J Natl Cancer Inst, 101, 1120-30.

Chaves EBM, Folgierini H, Capp E, et al (2012). Prevalence of abnormal anal cytology in women infected with HIV. $J$ Med Virol, 84, 1335-9.

Conley L, Bush T, Darragh TM, et al (2010). Factors associated with prevalent abnormal anal cytology in a large cohort of HIV-infected adults in the United States. J Infect Dis, 202, 1567-76.

Etienney I, Vuong S, Daniel F, et al (2008). Prevalence of anal cytologic abnormalities in a French referral population: a prospective study with special emphasis on HIV, HPV, and smoking. Dis Colon Rectum, 51, 67-72.

Ferrari A, Narasimhan K, Rahman I, et al (2008). Anal pap test in high-risk patients: a poor screening tool. Southern Med $J, \mathbf{1 0 1}, 1185-6$.

Gaisa M, Sigel K, Hand J, Goldstone S (2014). High rates of anal dysplasia in HIV-infected men who have sex with men, women, and heterosexual men. AIDS, 28, 215-22.

Ghosh I, Ghosh P, Bharti AC, et al (2012). Prevalence of human papillomavirus and co-existent sexually transmitted infections among female sex workers, men having sex with men and injectable drug abusers from eastern India. Asian Pac J Cancer Prev, 13, 799-802.

Kojic EM, Cu-Uvin S, Conley L, et al (2011). Human papillomavirus infection and cytologic abnormalities of the anus and cervix among HIV-infected women in the study to understand the natural history of HIV/AIDS in the era of effective therapy (the SUN study). Sex Transm Dis,
38, 253-9.

Lammé J, Pattaratornkosohn T, Mercado-Abadie J, et al (2014). Concurrent anal human papillomavirus and abnormal anal cytology in women with known cervical dysplasia. Obstet Gynecol, 124, 242-8.

MMWR Recommendations and Reports (2009). Guidelines for prevention and treatment of opportunistic infections in HIV-Infected adults and adolescents/recommendations from $\mathrm{CDC}$, the national institutes of health, and the HIV medicine association of the infectious diseases society of America. MMWR recommendations and reports. 58.

Mullins TL, Wilson C, Rudy BJ, et al (2013). Incident anal human papillomavirus and human papillomavirusrelated sequelae in HIV-infected versus HIV-uninfected adolescents in the United States. Sex Trans Dis, 40, 715-20.

Palefsky JM, Holly EA, Ralston ML, et al (1998). Anal squamous intraepithelial lesions in HIV-postive and HIV-negative homosexual and bisexual men: prevalence and risk factors. J Acquir Immune Defic Syndr Hum Retrovirol, 17, 320-6.

Piketty C, Selinger-Leneman H, Bouvier AM, et al (2012). Incidence of HIV-related anal cancer remains increased despite long-term combined antiretroviral treatment: results from the French Hospital Database on HIV. J Clin Oncol, 30, 4360-6.

Pittyanont S, Yuthavisuthi P, Sananpanichkul P, et al (2014). Prevalence of abnormal anal cytology in HIV-infected women: a hospital base study. Asian Pac J Cancer Prev, 15, 6405-9.

Siegel R, Naishadham D, Jemal A (2012). Cancer statistics, 2012. CA Cancer J Clin, 62, 10-29.

Solomon D, Nayar R (2004). The Bethesda system for reporting cervical cytology: definitions, criteria, and explanatory notes. $2^{\text {nd }}$ edition. New York: Springer-Verlag New York.

Suwannarurk K, Tapanadechopol P, Pattaraarchachai J, et al (2009). Hospital-based prevalence and sensitivity of highrisk human papillomavirus in Thai urban population. Cancer Epidemiol, 33, 56-60.

Thai National Aids Committee (2014). Thailand AIDS response progress report. national AIDS management center.

Zhang HY, Tiggelaar SM, Sahasrabuddhe VV, et al (2012). HPV prevalence and cervical intraepithelial neoplasia among HIV infected women in Yunnan Province, China: a pilot study. Asian Pac J Cancer Prev, 13, 91-6. 